ordinailes en bois. Pour chaque troncon du barrage, le gabarit comprenait 5 fermes semblables $\mathrm{BAG}$, espacées d'axe en axe de $3^{m} 05$, et reliées entre elles au moyen d'un contreventement an croix de St-André. Ces gabarits sont fixés à leur base sur la fondation générale du barrage, sur laquelle ils reposent au moyen d'un coussinet $\mathrm{C}$ à l'aval, et d'un embasement à cornière B à l'amont. Ces gabarits sont, en outre, reliés au pont de service par des tirants horizontaux $\mathrm{T}$. A l'intérieur de ces gabarits, on a fixé le coffrage en bois, puis on a procédé au coulage du béton. Celui-ci n'était pas pilonné, la quantité d'eau qu'il contenait étant telle que le tassement se faisait de lui-même par simple gravité. Une poutre longitudinale $\mathrm{A}$, indépendante des gabarits, et s'appuyant à ses deux extrémités sur la maçonnerie du barrage, sert momentanément de support aux gabarits lorsqu'on ellève leurs montants verticaux.

Les diverses pièces des gabarits et des coffrages ont été montées, démontécs et déplacées au moyen de 3 grues, d'un type spécial, se déplaçant sur le pont de service. Ces grues, qui sont représentées par la figure 2 , sont munies de 4 volées fixes, espacées de $3 \mathrm{~m} 05$, et capables de déplacer chacune $2270 \mathrm{kgs}$ à l'extrémité de la portée. Elles sont remorquées par less locomotives des trains qui amènent les matériaux, et leur's treuils de manceuvres sont actionnés au moyen d’air comprimé renfermé dans un réservoir $R$.

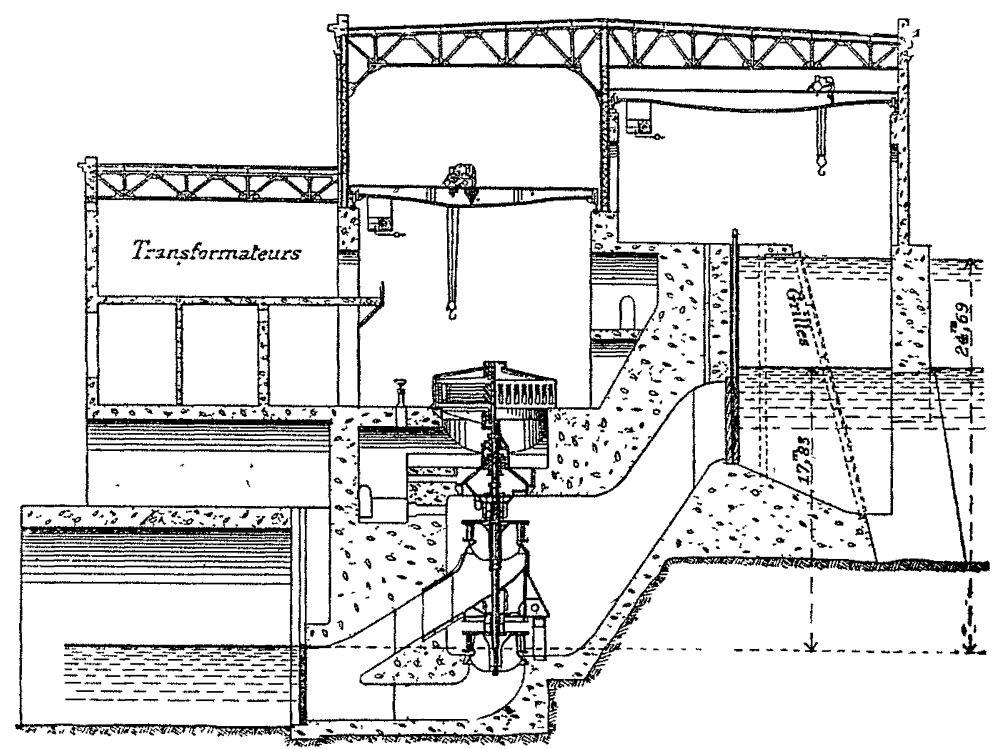

Fia. 3. - Coupe transversale de lusine de Mac Call Ferry.

Pour la construction de l'usine grénératrice, on s'est servi de 2 grues d'un type un peu différent, composées d'une volée mobile de $41 \mathrm{~m} 10$, et d'un bàti dont la hauteur totale atteint $38^{\mathrm{m}} 10$. Leur puissance est de 2270 kilogrammes qu'elles peuvent soulever $\dot{a} 27^{\mathrm{m}} 45$ au-dessus du pont de service précité.

Le barrage étant terminé sur toute son étendue, on procède à lobturation des vides de $12^{\mathrm{m}} 20$ ménagés entre chaque tronçon de la première partie de l'ouvrage dans le chenal de droite pour le passage de l'eau. Pour cela, on se sert d'un système de caissons mobiles, ou panneaux en bois rectangulaires, s'appuyant sur les deux troncons adjacents. Afin de bien assurer la liaison des deux parties, on avait ou soin de ménager des cavités dans les parois latérales des troncons primitivement construits.

On a fait emploi à ce barrage de joints de dilatation. Sur les deux parements, et aux points de jonction des massifs, on a ménagé des entailles, formant rainures, qui sont garniıs de papier groudronné. L'épaisseur de la rainure varie suivant la température du moment, et sa profondeur ne dépasse pas $0^{\mathrm{m} 90}$, limite extrême à laquelle on a admis que les changements de la température extérieure puissent produire un effet appréciable.
L'usine génératrice est construite tout en béton. Elle est prévue pour contenir 10 grosses unités génératrices à axe vertical de $13500 \mathrm{HP}$. Chaque turbine est double, centripete parallèle du système Francis, et est installée dans une chambre rectangulaire de $9^{\mathrm{m}} 14$ de largeur, $6^{\mathrm{m}} 70$ de longueur et $10 \mathrm{~m}$. de hauteur. Le diamètre de l'arbre est de $51 \mathrm{~mm}$. et celui des roues mobiles de $3 \mathrm{~m} 05$. Le graissage du pivot, qui supporte 150 tonnes, poids de la partie molile di groupe générateur, est assuré par des pompes lit huile actionnées par de petites turbines spéciales.

Chaque turbine est directement accouplée à un allerna. teur de $9500 \mathrm{kws}$, tournant à 94 tour's et produisant du courant trophasé à 11000 volts, 25 périodes. L'excitation est produite par deux dynamos de $1000 \mathrm{HP}$, tournant à 250 tours, et fournissant du courant continu à 250 volts.

Pendant la construction de l'usine, on a disposé des coffrages en bois, formant noyaux, à l'emplacement des conduites d'amenée et d'évacuation. On a coulé le béton tout autour, puis, l'on a enlevé les coffrages, ce qui a évitẻ lemploi de toute conduite métallique. Les conduites d'amenée sont protégées chacune par 4 grilles et par 4 vannes d'arrêt; elles sont de section rectangulaire et ont $9 \mathrm{~m} 14$ de largeur sur $3^{\mathrm{m}} 96$ de hauteur. Les conduites d'aspiration, également rectangulaires, sont quadruples, et ont $3^{\mathrm{m} 96}$ de hauteur sur $2^{\mathrm{m}} 28$ de largeur. Les grilles ont $3^{\mathrm{m} 05} \mathrm{de}$ largeur sur $3 \mathrm{~m} 3 \tilde{j}$ de hauteur; elles sont composées de harreaux plats de $114,3 \times 11 \mathrm{~mm}$., laissant $51 \mathrm{~mm}$. de vide entre eux. Les vannes ont $4^{\mathrm{m}} 88$ de hauteur.

La Susquehanna charriant beaucoup de glaces pendant l'hiver, on a aménagé une chambre d'eau devant l'usine. La prise d'eau se fait au travers d'un ouvrasco disposé normalement au barrage. A l'amont, cet ouvrage est constitué par un mur en béton reposant sur 3 arches de $20 \mathrm{~m} 70$ d'ouverture, avec piles de $2 \mathrm{~m} 44$. Ces arches sont à $0 \mathrm{~m} 61$ au-dessous des basses eaux, de manière à être toujours submergées. La crète de ce mur est à $6 \mathrm{~m} 70$ au-dessus du barrage. La partie aval est constituée par une estacade flottante s'appuyart sur des caissons en charpente.

Un atelier de fabrication du béton fut installé sur la rive droite; sa capacité de production était de $1500 \mathrm{~m}^{3}$ de béton par journée de 10 heures. Le barrage à lui seul a employé $133000 \mathrm{~m}^{3}$ de béton. La Compagńie établit aussi une petite cité ouvrière pour le logement des 1500 ouvriers qu'elle employait, la plupart d'origine italienne.

L. PALlORDET,
Ingénieur E. C.L

\section{LES VOIES NAVIGABLES EN FRANCE}

Uans son rapport sur les travaux publics en $1908 \mathrm{M}$. Audiffed, sénateur de la Loire, constate que des réclamations sont formulées sur tous les points du pays pour obtenir la construction de grandes voies navigables. C'est que partout, en effet, on comprend la nécessité de développer notre navigation intérieure comme l'ont fat les nations voisines : la Belgique, l'Allemagne, la Hollande, car il est incontestable que ces pays dolvent en grande partie leur prodigieux essor commercial ęt industriel a leurs réseaux de voies navl gables. La Suisse aussi se préoccupe très sérieusement de se créer un réseau navigable sous l'impulsion d'une vigoureuse campagne menée par M. R. Gelpke, ingénieur très connu, et d'autres notabllités baloises. Mis en gont par les beaux résultats qu'ils on obtenus en transperçant leurs montagnes de toutes parts, pour appeler sur leur territoire le trafic international des voies ferréess de liEurope centrale, les Suisses en sont arrivés à penser que Bâle pouvait devenir le nœud navigable de l'Europe centrale, avec, tout près. le lac de Constance, comme gigantesque bassin international, dans les entrepots duquel se manipuleraient et se distribueraient les produits divers du globe pour se déverser ensuite dans les différents centres commerciaux de leurope continentale.

A cet effel, l'état de navigabilité du Rhin serait poursuivi de, Strasbourg a Bale. par canal ou par rectification du fleuve, puis 
au lac de Constance. Un canal partirat de la pour rejomdre le Danube et desservir l'Autriche, la Hongrie, la Serbie, la Bulgarie et la Roumanie ; un autre pour rejoindre la vallée du Pó, desservirait l'Italie jusqu'à Venise sur l'Adriatique, et enfin une autre branche navgable viendrait jusquau lac de Genève drainer le trafic du versant françaıs. M. Gelpke, marchant déjà tout vıvant dans son rève étollé, conclut en s'écriant :

Le proyramme de la créalion d'un réseau navigable avec la Suisse comme centre est tracé. On travaille dé tions de cetle nouvelle et puissante cuvre de civilisation.

Simultanément au projet de $M$. Rud Gelpke, un autre ingéneur hydraulıcien. l'itahen Cammada, étudie un projet de construction d'un gigantesque canal partant de Génes et traversant la Lombardie, puis les Alpes et la Suisse, pour atteindre le lac de Constance.

Ce canal, d nne longueur de 591 kilomètres, et d'un gabarit correspondant aux bateaux de 600 tonnes en usage sur le Rhin, serait une vole de communication directe de Rotterdam et du bassin de la Rulr avec Gênes. Le trafic annuel de ce canal est estrmé à 15 millons de tonneaux ; son bief le plus élevé est au Splugen, a 1217 metres daltılude, et son coùt est provisoirement évalué à 400 millions.

Certes, comme le falt justement remarquer M. Arnodin dans un interessant rapport présenté à la Cliambre de Commerce d'Orleans. de tels projets amèneront sur les lèvres de la plupart des Francus un sourıre d'incrédulité ; mals n'avons-nous pas déjà sour' de la meme façon lorsquion nous a parlé pour la première fors du Gothard et du Simplon, qui aujourd'hu nous enlèvent un trafic qui, normalement, devrait passer par notre pays ?

Nos concurrents savent bien que les deux mers où s'exerce avec le plus d'intensilé la concurrence des nations sont, d'une part, le canal qu sépare l'Europe de l'Angleterre, ou se trouvent groupis dans un petit espace des ports comme Hambourg, Rotterdam, Anvers. Jondres. Douvres, Southampton, Dunkerque, Le Havre, Roven mome, et d'autre part la Méditerranée, sur les rives de laquelle sunt silués Barcelone, Alger, Marseille, Gênes, et où débouche lo canal de Suez, avec tout au fond Constantinople et la mer Notre.

Cette situation leur semble de nature à justifier tous les sacrifices pour s'assurer les voles de transport terrestre entre ces deux champs d'activité. Il faut reconnaitre cependant qu'un réseau navigable international convergeant vers Bâle n'est certainement pas la route naturelle, c'est-à-dire la plus pratıque et la plus rcunomique.

Il faut donc conclure avec M. Irnodm qu'un tel projet ne peut porter de fruits que sil est favorisé par l'apathie et le manque de clairvoyance du vousm, nous Français, dans la curconstance.

Quelles sont donc les voles navigables logiques?

II Arnodm nous le démontre d'une facon ingéneuse. - Supposons, dit-ll, toutes les mers entourant l'Europe se gonflant parallèlement comme par un immense ras de marée. - Lorsque ce gonflement gigantesque aura attemt $378 \mathrm{~m}$. 50, la mer du Nord couvrira entièrement la Hollande, les Flandres et les basses terres du Nord Les eaux se confondront avec la Manche dans la vallée de la Seme, et le flot commun sera porté vers le canal de Bourgogne en remontant la vallée de l'Yonne ; alors que, par un mouvement parallèle, la Méditerranée remontera la vallée du Rhone et celle de la Saone, pour rejoindre le flot venant du Nord. La jonction se fera au point de partage des deux versants, vers Poully-en-Auxors (altutude 378.50) sur le canal de Bourgogne.

La mer du Nord remontant par la vallée du Rhin dépassera Strasbourg et Bale (altutude 252 mètres), approchera du lac de Constance (altitude 399 mètres), mais ne pourra pas aller plus loin, barrée qu'elle sera par le massif infranchissable des Alpes.

Le flot du Nord sera donc obligé pour gagner la Méditerranée, de venir passer par la trouée de Belfort, entre les Vosges et la chaine du Jura, pour atteindre la Saone se joignant au Rhône ¿ Lyon (altitude $160 \mathrm{~m} .54$ ).

Simultanément l'Atlantique, remontant par la vallée de la Loire, atteindra d'une part Nevers, suivra la dépression empruntée par le canal du Centre et aura déjà rejoint le flot méditerranéen vers l'embranchement du Creusot (altitude 301 mètres 55), mettant ansi 76 mètres 55 de hauteur d'eau sur le col de partage des versants de la Loire et de la Saone, un peu en amont de Lyon et a faible distance en aval de St-Jean-de-Losne, où se trouve l'embouquement des voies navigables de l'Est allant vers Mulhouse et Bâle, ou vers Nancy, Strasbourg, ou vers Nancy, Trêves, Coblentz, Rolterdam.

D'autre part. l'Alantique. poursuivant son chemin dans la vallée de la Lorre, arrivera près de Sant-Etıenne (altıtude 516 mètres), pendant que la Méduterranée amenée par le Rhơne, remontera le Gier également vers St-Etienne où il s'en manquera seulement d'une centaine de mètres pour qu'une deuxième soudure puisse etre réalisée sur ce point.

Or le Gier a son confluent avec le Rhône un peu en aval de Lyon. C'est donc dans la région qui avoisine Lyon et Vienne que se ferait la jonction des trois mers, qui baignent l'Europe centrale, si le gigantesque ras de marée, supposé par M. Arnodin, venalt a se produire el cela en dehors des travaux des hommes, c'est-à-dire par la voie naturelle.

De tout ce qui précède il faut conclure, sans crainte de se tromper, que le seul et véritable noeud navigable de-l'Europe centrale est Lyon et non Bâle, comme voudrait le faire admettre M. l'ingenieur R. Gelpke, poussé par un sentiment de patriotısme peutêtre un peu excessif en la matière.

Nous croyons avoir démontré que le noud navıgable de l'Europe centrale est. Lyon et non pas Bàle. De là découle logiquement que la grande vole internationale fluviale faisant suite au canal de Suez pour gagner le nord de I'Europe est Marsenlle. Lyon. Paris, Rouen, Le Havre ; celle pour gagner l'Atlantique : Marselle, Lyon, st-Etrenne, Roanne, Orléans, Nantes, St-Nazaire, ou plus au sud : Marseille, Beaucanre, Toulouse (par le canal du Midi). Bordeaux.

Tous ces tracés naturels ne nécessitent pas de travaux gigantesques, ils traversent des régions tempérées, fertiles et peuplées, alors quau contraire les voles préconisées par les Sulsses et les Itahens, passent ì de hautes altitudes, loin des populalions nombreuses, et chaque année la navigation y serait longuement interrompue par les glaces.

M. Arnodin fait aussi remarquer justement que Lyon est en facle communication avec le Rhin, Rotterdam et les provinces rhénanes par tross voies : la première Coblentz, Trèves, Metz, Nancy, Moselle canalisée, canal de l'Est et la Saone - la seconde Strasbourg, Nancy, canal de la Marne au Rhin, canal de l'Est, et la Saône - la troisième Mulhouse, St-Symphorien, canal du Rhơne au Rhin et la Saône, qui dessert également Bale.

D'autre part, le port d'Anvers est relié à Nancy, par sulte à Lyon et i Marsellle, par Charlerol, Namur, la branche nord du canal de l'Est : Anvers peut aussi atteindre Lyon et Marseille par St-Quentin et Paris.

Dunkerque peut également venir à Lyon, solt par Nancy, le canal de St-Quentm et le canal de la Marne au Rhin, soit par l'Oise, Parıs et la Bourgogne. Enfin, il est faclle d'établir une voie navigable du lac de Genève à Lyon, par le haut Rhone, pour desservir la Susse française (proposition Baudin).

De toutes ces considérations ressort nettement que Lyon est beaucoup mieux plaćé que le lac de Constance pour centraliser dans de vastes magasins et distribuer dans l'Europe centrale les produits du Nord et de l'Extrême-Orient.

La navigation de Bordeaux peut facilement attemdre Lyon par le canal du Midi et la vallée du Rhone.

Nous aurions ainsi en France $: 1^{\circ}$ la vole internationale princıpale : Marseille, Lyon, Parıs, Rouen, Le Havre, la mellleure et la plus courte de toute l'Europe, la moins accidentée et celle qui passe dans les régions les plus populeuses et les plus tempérées ;

$2^{\circ} \mathrm{La}$ voie secondaire . Nantes. Orléans, St-Etrenne, Lyon, Marselle, également très importante ;

$3^{\circ}$ La voie tertnaire : Bordeaux, Toulouse, Montpellier. Beancarre, Marseille ou Lyon, Paris et Nord.

La France, dans de telles conditions, serait le pays le mieux oufıllé du monde entıer comme moyens de transport, puisque nous avons le mellleur réseau de routes qui existe, et que nos Compagnies de chemins de fer ne craignent, à l'heure actuelle, aucune comparasson au point de vue du matériel et de l'organisation.

Que faut-l farre pour arriver à ce magnifique résultat ${ }^{?}$ Il s'agit tout simplement d'envisager sérieusement la question et ensuite détudier les voles et moyens pour arriver à une solution générale qui n'est pas au-dessus de nos forces.

Pratiquement voici ce qu'il convient de farre sans tarder

Il faut tout dabord se hater d'ouvrir le canal latéral au Rhone, y compris le raccordement de Marseille au Rhone actuellement en construction. Nous savons mieux que personne, nous qui habitons la vallée du Rhone, que sur ce fleuve ne peut s'effectuer qu'une navıgation précaire ; malgré tous les progrès réalisés, le Rhone reste déserté de la batellerie, car le courant y est si impétueux que tout bateau de canal en bois descendu conte autant a remonter que vaul la coque.

The la de tristes conséquences que ne manque pas de relever 
lauteu que nous avons cité plus haut : tout a abord la marchandise se trouve grevée de frais tels que le transport par batellerie est aussi onéreux que celui par chemin de fer et devient souvent trop élevé pour certaines marchandises qui préfèrent la voie de mer, malgré tous ses inconvénients et ses risques.

De là il résulte éncore que notre vallée du Rhône, qui devrait être le Gibraltar d'eau douce pour communiquer d'une mer à l'autre, ne réalise pénblement qu un tonnage annuel de 300.000 tonnes, alors que la vallée du Rhin, certamement moms bien douée par la nature, a vu transiter en 1905 plus de 58 milhons de tonnes.

Troisième résultat extraordinaire : les produits d'Orıent à destination de Strasbourg et de lAlsace vont passer par Gubraltar, contournent l'Espagne et 1a France en navire; et vont sembarquer a Rotterdam sur les bateaux du Rhin, qui remontent jusqu'à Strasbourg, alors que le vaisseau les apportant a souvent touché Marseille, et que Strasbourg' est à peu près aussi éloigné de Rotterdam que de Marseille. Cette circumnavigation est rendue nécessaire parce que le Rhin est économiquement navigable, alors que Ie Rhône ne l'est pas : de plus, les relations avec la douane française sont hérıssées de telles difficultés quon cherche tous les moyens d’éviter le passage des marchandises sur notre territoire.

Outre le canal latẻıal au Rhóne, il faut prolunger le canal de la Loire en aral jusqu'a Nantes, ou tout au moms jusqu'ì la rencontre avec la Loire navigable ; en amont il faut le conduire jusqüä St-Etrenne. d'où il descendratt vers Lyon par le canal du Gier, en traversant une contrẻe industrielle, dont la prospérité est enrayée. parce qu'elle se trouve encore aujourd'hui dépourvue de communications pratiques par eau.

Il faut enfin améliorer les conditions de navigabilité du canal du Mudi et du canal latéral de la Garomme, qui devrazent pouvoir conduire de Bordeaux à Marseille, mais qui se terminent en culde-sac au Rhône. à Beaucaire, sans atleindre Marseille.

Ces travaux ctant faits, la France dotée d'un réseau navigable homogène, aboutissant sur toutes les mers aux ports princıpaux de l'Europe continentale, qui sont Rotterdam. Anvers, Dunkerque pour la mer du Nord, Le Havre, Rouen pour la Manche ; Nantes, St-Nazaire pour lAtlantique nord ; Bordeaux pour IAtlantique sud : Marseille pour la Méditerranée, pourra facilement échanger ses prodults contre ceux du monde entier et servir de voie de transit aux nations voisines. Il est faclle de comprendre que le trafic ainsi créc dépassera de beaucoup celur de quinze milhons de tonnes prévu pour le tracé Suisse, et que, comme conséquence, la grande industrie sera retenue sur notre territoire : or, à la présence et à la prospérité de celle-ci est liée la prospérité de la nalion tout entière, comme le confirme l'exemple des Etats-Unıs et de l'Allemagne.

En examinant sér ieusement les considéi ations, que nous avons fart valour dans notre exposé. il y a lieu de s'étonner de notre extraordinarre apathe nationale; nous ne voulons pas profiter des avantages que nous a si libéralement concédés la nature, et nous restons absolument rebelles à l'étude des grands problèmes économiques qui peuveut assurer la prépondérance et la richesse de notre pays.

Il ne faut pas se dissimuler que nous sommes actuellement en décadence, quoiqu'en disent certains économistes superficiels. La preuve est malheureusement facile d administrer : sans remonter au XVIII ${ }^{\circ}$ siècle, où nous.étions sans conteste la première nation du monde en toutes choses, il suffit de se reporter à une trentaine d'années en arrière pour voir clairement les échelons que nous avons descendus.

Pour le commerce général, exportations et importations, nous étions en $18 \%$ la deuxième valion du monde immédiatemet après l'Angleter'e. Nous arrivons aujourd'hui à peme à égaler la Hollande, bien loin derrière 'l'Allemagne et les Etats-Unis.

Pour la marne de guerre nous étions la deuxic̀me nation; nous arrivons maintenant la cinquième, après le Japon.

Pour la marine marchande, nous étions deuxième; nous sommes aujourd'hui septième, après I'Italie.

Pour les industries chimiques, qui ont pris naissance chez nous, nous sommes it Theure actuelle de beaucoup dépassés par l'Allemagne.

Pour les arts mécanrques, nos métıers, dont Jacquard est pourtant linventeur, nous viennent pour la plupart d'Angleterre ou d'Allemagne; nous achetons nos wagons en Belgique, nos locomotives en Amérique et en Allemagne, et nous étions autrefois les fournisseurs de ces nations, dont nous sommes actuellement les tributaires.
Nuus acceptons béllévolement et avec grande mdıltérence cotte dimulution de prestrge el d'alfarres. La France est riche, disons. nous. Gest vrai et nos. grosses disponibulutés. servend a tontes. les nations, a qui nous prètons notre argent avec une singuhère faci litê au lieu de l'employer chez nous. De plus' comme le fai justement remarquer $M$. Arnodin, la France contemporame nest. elle pas dans la situation d'une maison de commerce, naguèn prospère, qui ne renouvelle pas son outillage, qui restreint son chiffre daffaires et qus voit de ce fait ses disponıblités grossir plus vite que celles du concurrent qui augmente son outillage et ses approvisionnements pour ar̉river plus facilement à annihiler la coneurrence. L'un vit sur les bénéfices passés ; lautre recherch de plus grands bénéfices dans l'avenur. et ce fassant, - il répan davantage de prospérité autour de lur.

Quel remède faut-il apporter à notre état de décadence natıonale; presque de décrépitude ? Il faut insister auprès des pouroip publics afin qu'lls se préoccupent enfin d'outiller utllement notr pays pour qu'll solt en mesure de lutter moins désavantageusement contre ses rivaux.

Beaucoup de rétrogrades vont s'écrier qu'il faudra trop dargent pour exécuter les compléments indispensables̀ de nos voles nav. gables. Il est facle de répondre que d'autres pays moins ruches que nous le font à notre détriment et la plupart avec l'argent de nolre épargne. Il est done urgent de canaliser cette épargne vers nos travanx d'utılite pratıque au heu de la laisser s'employer pour ceux des autres.

On a évalué que la perté fate a la fortme francause par le détournement de trafic, créé par le Gothard et le Simplon, peut atteindre 200 millions par an. C'est à peu près la somme qu'll falldrait pour farre un canal de Nantes à Briare ef le tiers de celle nécessaire pour doter notre vallèe du Rhône dun canal capable de nous ramener. el au-delà, tout le trafic qui nous est détourné par ces voies concurrentes. La dépense seralt donc promptement amortie, de plus, tous les économistes admettent qu'une nation qui dépense son argent chez elle en ouvres d'utilıté publique s'en. richit toujours lorsque le travail est fait par ses nationaux ot qu'elle acquière comme augmentation de rzchesse nationale le pro. duit du nouvel outillage qui vient d'etre créc par ses capitaux propres.

Nous devons mamtenant nous résumer et nous ne nanquerons pas de le faire brièvement.

La grande industrie, qui engendre la prospérnté d'un pays, ne peul s'établır que sur les grandes routes internationales desservies par la roie d'eau et en contact avec le chemm de fer ; la première almmentant les usines en matuères premıères et pondé. reuses, par gros groupements qui se pretent à l'ćconomie; lè second servant plùs spécialement au personnel et aux marchandises ourrées et à celles dont la valeu marchande permet de subir des frais de transport plus élevés.

L'organisation de nos chemins de fer u est plus adéquate aux besoins à desservir surtout parce quils sont encombrés de transports de matières qui devraient normalement aller à la vole d'eàu beaucoup plus économique ; matières que les chemins de fer transportent sans bénéfice, souvent même à perte, en couvrant le déficit par une élévation de tarif sur d'autres articles.

La crise des chemins de fer, qui frappe tous nos réseaux, sauf peut-être le Nord qui est doublé d'une voie d'eau, crise que subis: sent aussi les nations voisines, est la preuve de l'insuffisance de la voie ferrée. Le chemm de fer isolé, ciest-à-dire non secomdé par une voie navigable, demeure impuissant à desservir un mout vement industriel el commercial qui doit certainement devenir de plus en plus intensif.

Enfin, il est de toute évidence que toutes les nations qui se seront au préalable outillées en voies navigables prendront ou conserveront l'avance sur leurs concurrentes en vue du développement du mouvement commercial et industriel a venur.

C'est pour cela qu'asquiesçant à un vœu émis récemment par la Chambre de Commerce d'Orléans nous croyons qüll est du devoir de tous les Français soucieux de la prospérité nationale d'in sister auprès des pouvorrs publics pour qu'jls hâtent l'établisse. ment d'une voie navigable dans la vallée du Rhône et dans la vallée de la Loure, car, faule de ces deux troncons dimportance primordiale, les sacrifices consentis jusqu'à ce jour pour la navigation intérieure et marıtime ne sont qu'incomplètement productifs et le pays reste insuffisamment armé pour les luttés économiques de l'avenir.

Claude MaYE

Docteur en droil. 
Pour fixer plus complètement les adées de nos lecteurs et résumer nos considérations, nous croyons devoir mettre sous leurs yeux $1^{\circ}$ les principaux itinéraíres navigables, reliant la Méditeryeux . arec l'Océan Atlanlıque, la Manche et la mer du Nord, avec noud central à Lyon; $2^{\circ}$ les distances comparées des voies navigables dont nous avons parlé.

linnéraires principaux (Alttude maxima : $378 \mathrm{~m}$.)

\section{EN France}

I $\mathrm{o}^{\mathrm{r}}$ Narselle, Beaucaire, Toulouse, Bordeaux. $2^{\mathrm{e}}$ Marselle, St-Etienne, Roanne, Orléans, Nantes. St-Nazaire 3e Marseille, Lyon, Parıs, Rouen, Le Havre.

\section{Vers la Belgique}

Le Marselle, Lyon, Paris, St-Quentm, Mons, Anvers.

III Vers la Hollande

$5^{e}$ Marselle, Lyon, Nancy, la Moselle, Coblentz, Cologne, Rotterdam.

IV Ve Vers L'ALLEMAGNe

6e Marselle, Lyon, Nancy, Strasbourg, le Rhin.

Ve Vers la Suisse

$i^{e}$ Marselle, Lyon, Dôle, Mulhouse, Bâle, Constance.

\section{Dislances comparées}

Gines a Rolterdam, par le lac de Constance et Bale, $1.612 \mathrm{kll}$. llarselle à Rotterdam, par Strasbourg, 1,730 kil. Narselle it Rotterdam. par Nulhouse, 1,597 kil. Marselle à Rotterdam, par Nancy, la Moselle. Coblentz, $1.550 \mathrm{k}$. Narselle au Havre, pai l.yon-Paris, 1,38: kil. Marselle a Rouen, par Lyon-Paris, 1,261 kil.

\section{Coneessions des Forces hydrauliques sur les Cours d'eau faisant partie du domaine public}

Le jeudı 9 avril Igo8, la Chambre de Commerce de Grenoble s'est réunie dans son hôtel, sous la présidence de M Brenier, président. M. Marius Viallet a donné lecture du rapport suivant que nous croyons du plus haut intérêt de placer sous les yeux de nos lectleurs.

\section{MESSIEURS,}

"La mise en valeur des grandes forces hydrauliques prend dans les Alpes une extension considérable, qui se traduit par une activité industrielle sans précédents dans les annales du Dauphnné et de la Savoie; c'est amsı que le capital actions ou obligations des sociétés constituées dans notre région au cours de ces dernières années, basées sur l'utilisation de la houlle blanche, dépasse cent millions de francs.

"Dans le bassin de l'Isère, l'un des plus riches de France et dont l'ensemble des forces hydrauliques, évalué à un million de chevaux en eaux moyennes, constitue une véritable richesse nationale, l'on ne comptait pas moins, au commencement de 1906, d'une cinquantaine d'installations, disposant chacune de plus de 100 poncelets en basses eaux et dont la puissance totale utilisée variart, suivant les époques de l'an'née, de 80.000 à I 70.000 chevaux (I).

"Ces usines, dont le nombre croît rapidement, se sont toutes installées sur des cours d'eau non navigables, ni flottables; le domaine public, représenté dans notre région par la nivière d'Isère, d'Aigueblanche à Valence sur une longueur de 217 kilomètres, est, jusqu'ici, resté intact. Il n'en sera pas toujours ainsi : déjà une demande en concession, déposée au commencement de l'année 1906, sur laquelle il n'a pas encore

(1) Ministère de l'Agriculture, direction de l'hydraulique, service des lilsere, des grandes forces hydrauliques - Region des Alpes, bassin de Plsère, M. R DE la BRosse, ingenieur e 1 chef des Ponts e: Chaussées.
Fascicule $i_{2}$, page 43 . été statué, vise l'aménagement de la Basse-Isère, à Châteauneuf (Drôme), en vue de l'établissement d'une usine hydroélectrique.

"Nous ne voyons, en ce qui nous concerne, que des avanlages à la prompte mise en valeur des forces hydraulıques, où qu'elles se trouvent, car la houille blanche ne se capitalise pas : c'est une rente perpétuelle, dont les arrérages sont servis au jour le jour et se prescrivent sans délais.

"Notre désir de vour utiliser les forces vives des cours d'eau ne doit cependant pas nous faire oublier les précieux avantages que la collectivité peut retirer des rivières, ces chemins qui marchent et qui doivent rester à la disposition de tous.

"L'Isère, en tant que route nationale, n'est pour ainsi dire pas fréquentée : c'est à peine si l'on peut enregistrer le passage à Grenoble d'une centaine de bateaux, d'une portée totale de 7 à 8.000 tonnes, et de quelques trains de bois de valeur minime; malgré le peu d'importance de cette circulation, notre Chambre de Commerce ne peut se désintéresser des conditions mises à l'occupation éventuelle de cette partie de notre domaine national, car, si rien n'a encore été fait pour améliorer la navigabilité de l'Isère, de Grenoble au Rhône, tous les éléments du problème réunis paraissent offrir des gages certains de réussite : le débit, qui ne descend jamais au-dessous de 100 mètres cubes par seconde, au confluent du Drac, c'est-à-dire aux portes de Grenoble, et la dénivellation de 100 mètres sur un parcours d'environ 120 kilomètres, dont on pourrait tirer parti au profit de la navigation elle-même, permettent d'entrevoir des solutions pratiques et économiques. Quant au trafic, il est dès ce jour très largement assuré : a la descente par les produits fabriqués et à la remonte par les matières premières; à eux seuls, les ciments des environs de Grenoble et les anthracites du bassin de La Mure représentent un tonnage de près de 500.000 tonnes, dont une grande partie emprunterait avantageusement la voie d'eau.

"Vous le voyez, Messieurs, l'Isère peut être dans l'avenir un affluent bien précieux pour le Rhône, et Grenoble port fuvial n'est pas un rêve aussi chimérique que certains se l'imaginent. Lorsque ce rêve aura pris corps, nos industries régionales disposeront des deux éléments les plus indispensables à leur prospérité et à leur développement : la force et les transports à bon marché.

"Ce que nous venons de dire de l'Isère s'applique à beaucoup de rivières qui, pour des causes diverses, n'ont pas encore été utilisées autant qu'elles auraient pu l'être; pour permettre aux collectivités de veiller à ce que rien ne vienne compromettre l'avenir, il importe de donner une large publicité à toutes les demandes en concession de forces hydrauliques dépendant du domaine public, et de l'étendre surtout de façon à ce qu'elle touche tout au moins les plus directement intéressés. Aussi, rous proposons-nous d'émettre le vœu suvvant, étudié en vue de donner satisfaction à ce desideratum. dont l'importance ne saurait vous échapper :

"Les enquêtes prescrites par la loi du 8 avral 1808 et réglémentées par le décret du $I^{\mathrm{er}}$ aon̂t 1905 , portant sur les demandes en autorusation d'usines hydrauliques à établir sur les cours d'eau navigable, seront ouvertes, non seulement dans les communes oì les travaux devront être exécutés, mais encore dans tous les départements trazersés par la rivière visée à la pétution;

"A cet effet, un dosszer complet des pièces de l'instruction sera déposé à la Préfecture de chacun de ces départements et les Chambres de Commerce intéressées en recevront un exemplaire dvec invitation à formuler un aris motivé sur la demande présentée ".

- Après discussion, la Chambre approuve les conclusions ci-dessus et les convertit en délibération. Elle ordonne l'impression du rapport et son envoi à MM. les Ministres du Commerce et des Travaux publics, à M. l'Inspecteur général des Ponts et Chaussées (de la $7^{\text {me }}$ Inspection) et à $M$. l'Ingénjeur èn chef du département de l'Isère. 